\title{
Impact of Coordinated-Bilateral Physical Activities on Attention and Concentration in School-Aged Children
}

\author{
Heidi Buchele Harris, ${ }^{1}$ Kai Schnabel Cortina, ${ }^{2}$ Thomas Templin, \\ Natalie Colabianchi, ${ }^{1}$ and Weiyun Chen $\left(\mathbb{1}^{1}\right.$ \\ ${ }^{1}$ School of Kinesiology, University of Michigan, Ann Arbor, MI 48109, USA \\ ${ }^{2}$ Department of Psychology, University of Michigan, Ann Arbor, MI 48109, USA \\ Correspondence should be addressed to Weiyun Chen; chenwy@umich.edu
}

Received 30 August 2017; Revised 25 October 2017; Accepted 24 April 2018; Published 28 May 2018

Academic Editor: Haichun Sun

Copyright (c) 2018 Heidi Buchele Harris et al. This is an open access article distributed under the Creative Commons Attribution License, which permits unrestricted use, distribution, and reproduction in any medium, provided the original work is properly cited.

\begin{abstract}
Purpose. This study examined the effects of 4-week, daily 6-minute coordinated-bilateral physical activity (CBPA) breaks in classroom on attention and concentration in school-aged children. Methods. Participants ( $n=116)$ in fifth grade from two elementary schools were assigned to three groups: two intervention groups $(n=60)$ and one control group $(n=56)$. All three groups were preand post-tested with the $\mathrm{d} 2$ Test of Attention ( $\mathrm{d} 2$ test). One intervention group $(\mathrm{n}=31)$ took part in six minutes of daily classroombased coordinated-bilateral physical activity (CBPA) break for four weeks. Another intervention group $(\mathrm{n}=29)$, the Fitbit Only (Fitbit-O), wore Fitbits per day during a school, five days per week for four weeks without CBPA breaks. A $2 \times 3$ ANOVA was conducted, followed by the post hoc comparisons. Results. The CBPA showed significant increases in processing speed $\left(F_{1}=6.876\right.$, $p=.010)$, focused attention $\left(F_{1}=10.688, p=.002\right)$, concentration performance $\left(F_{1}=26.46, p=.000\right)$, and attention span $\left(F_{1}=14.090\right.$, $p=.000$ ) over the control, but not in accuracy (Error \%). The CBPA showed significant improvement in concentration performance $\left(F_{1}=24.162, p=.000\right)$ and attention span $\left(F_{1}=6.891, p=.011\right)$, compared to the Fitbit-O. No significant changes in all five attention parameters were found between the Fitbit-O and the control. Conclusion. It was concluded that daily brief coordinated-bilateral activities can improve attention and concentration in fifth-grade students over the course of four weeks.
\end{abstract}

\section{Introduction}

Studies have shown that physical activity (PA) is positively associated with academic performance [1-11]. Individuals are more likely to have better mental focus and concentration when engaging in structured physical activity [11-18]. However, children are sitting in the classroom for most of a school day [1-3]. A prolonged sedentary behavior not only does reduce children's attention to instructional tasks and their sustained focus on the task engagement, but also prevents children from meeting a recommended daily amount of moderate-to-vigorous physical activity (MVPA) [11-20]. To address the critical concerns, classroom-based PA breaks have been increasingly used as an effective intervention strategy to improve students' academic achievement, academic behaviors, and executive functions [11-20].
A handful studies have examined the impact of classroombased PA breaks on attention and concentration quantitatively and qualitatively $[3,19-28]$. The studies have shown that the classroom-based PA can improve children's attention and concentration [19-24]. In a study of implementing daily 10-minute physically active academic lessons (Energizers) to 62 third- and four-grade students for 12 weeks, Mahar et al. [23] found significant increases in on-task behaviors (i.e., appropriate verbal or motor behaviors) from pre-Energizers treatment to post-Energizers treatment. Additionally, students in the energizer group took a significantly higher number of steps than the control group by the end of each day [23]. Similarly, in a study of implementing a daily 1015 minutes physically active lesson (Texas I-CAN) to thirdgrade students, researchers observed each student's time-ontask (TOT) within 15-minute before and after the physically 
active lesson and the inactive lesson (control) for 16-22 times [24]. The study found that the students' TOT was increased slightly from before to after the active lesson, while the students' TOT was decreased significantly from before to after the inactive lesson.

Although classroom-based PA studies have documented a willingness of teachers to use physically active lessons, some barriers such as added training, extra preparation time, and additional demanding on knowledge base for implementing physically active lessons may lead to difficulties for scalable and sustainable implementation [3, 22, 25]. To reduce additional workload for the teacher to implement the classroom-based PA breaks, Howie et al. [26] examined how long the classroom PA breaks should last using a four-tiered approach of 10 -minute sedentary breaks versus 5,10 , and 20 minutes of classroom-based PA breaks. They found that all PA breaks showed statistically significant improvements in timeon-task (TOT) with the most pronounced effect occurring with the 10-minute PA breaks compared to the sedentary break. Further, Ma et al. [27] found that four minutes of inclass high-intensity PA breaks increased selective attention and reduced errors assessed with $\mathrm{d} 2$ Test of Attention in 9to 11-year old children.

More recently, researchers have examined the effects of coordinated-bilateral exercises on attention and concentration in school-aged children [2, 28]. Coordinated-bilateral exercises are specifically designed to use both sides of the body and more body parts simultaneously to perform bilateral movements while crossing the midline of the body $[2,28]$. Coordinated-bilateral movements engage both hemispheres of the brain and may facilitate cognitive development of cerebellum and prefrontal cortex [29, 30]. Budde et al. [2] found that the coordinated-bilateral exercise in physical education (PE) lessons led to significant improvements in children's attention assessed with d2 Test of Attention. Similarly, Schmidt et al. [28] examined 90 fifth-grade students' attention performance tested with the revised version of the $\mathrm{d} 2$ Test of Attention (d2-R) before, immediately after, and 90 minutes after an acute bout of coordinative exercise in PE. They found that the coordinated exercise significantly increased children's attentional performance $90 \mathrm{~min}$. after cessation, but not immediate. Furthermore, in a systematic review of PA interventions, Van der Fels et al. [31] suggest that the short burst of fine and gross motor coordinatedbilateral PA can improve attention, processing speed, and focus [31]. However, the previous studies have limited to examining one time effects of coordinated-bilateral exercise on attention and concentration, the key component of executive functions. Therefore, this study aimed at examining the effect of 4-week, daily 6-minute coordinated-bilateral physical activity (CBPA) breaks after 20 minutes of sedentary on attention and concentration in school-aged children. This study hypothesized that students in the CBPA breaks would show a greater increase in their attentional performance measured with the $\mathrm{d} 2$ Test of Attention compared to the comparison groups. The significance of this study was that the intervention was designed for the classroom teachers to instantly engage all students in CBPA breaks without any additional preparation by showing 6-minute CBPA videos.
Therefore, the study intervention strategy would be feasible and scalable for classroom teachers to implement.

\section{Methods}

2.1. Design. This study used a quasi experimental design to assign one elementary school to the intervention school and another to the control school. In the intervention school, one fifth-grade class was assigned to the CBPA group $(n=31$, 17 boys versus 14 girls), and another fifth-grade class was assigned to Fitbit Only (Fitbit-O) group ( $n=29,19$ boys versus 10 girls). In the control school, two fifth-grade classes were assigned to the comparison group ( $n=56,21$ boys versus 35 girls). This study was conducted over the course of seven weeks. The first week was used for recruitment. The students took the $\mathrm{d} 2$ Test of Attention at baseline during the second week and took the test again immediately after the 4-week intervention during the seventh week.

2.2. Participants. Approval was obtained from the University Institutional Review Board- (IRB-) Health Sciences and Behavioral Sciences (HSBS) (HUM00102732). The signed consent forms were obtained from the parent/guardian of 116 students who were recruited from four, fifth-grade classes at two elementary schools. Also, written assent forms were gathered from the students prior to pre- and post-testing. Two elementary schools with a racially and socioeconomically diverse student population were selected based on their fiscal and racial similarities and their voluntary participation in this study. $43 \%$ of students in the intervention school and $37 \%$ of students in the control school received free or reduced lunch. In the intervention school, $60 \%$ of the participants self-identified a race other than white, with 30\% African American. At the comparison school, 48\% self-identified a race other than white, with $19 \%$ African American.

\subsection{Treatments}

Fitbits. Students in the CBPA and the Fitbit-O groups were given Fitbits Charger HR to wear from Monday morning (as they arrived at the classroom) until Friday afternoon, whereupon they put the Fitbits in the charging station before they left for the weekend for four weeks, except in cases when they might get wet. Students were able to check their own steps taken, real time heart rate, distances traveled, and calorie burned on the Fitbit. Students were encouraged to keep track of their own information and to set goals for themselves, while teachers were given reports of the classroom average steps and activity minutes each Monday by the investigator. In contrast, the students in the control group wore plastic wristband for four weeks.

Coordinated-Bilateral Physical Activity (CBPA). Students in the CBPA group participated in 6-minute repetitive coordinated-bilateral motor movements while following 6minute video instructor's CBPA once a day after they had been sitting for 20 minutes of a class instruction for five days per week, over four weeks. The CBPA videos were deliberately designed to emphasize coordinated exercises which use bilateral body movements and to be progressive 
in difficulty and speed [32]. The CBPA videos started slowly and rhythmically during the first week and were gradually done faster and more complicated in weeks of 2 and 3. During the week 4 , the levels of difficulty and speed were further increased. Motor movements were rhythmically repeated eight, four, and two first in unison and then in opposite directions. Examples of motor movements were making figure eights by simultaneously pairing arm movements in the same direction, by changing the direction, and by having the arms go in opposite directions. Then, motor skills utilized the entire body. Children went from a split to a squat stance, first in unison then in opposite directions, so the video instructor jumped sideways, and the participants were encouraged to squat. The teacher reported missing 3 days of CBPA. The Quick Time videos were labeled Day 1 through Day 20. The control group was not asked to make any changes to their normal school day beyond taking the additional pre- and post-d2 Tests administered to all three groups.

Comparison Group. The students in the comparison group followed their school scheduled academic instruction periods. In other words, the comparison group was not asked to make any changes to their normal school day beyond taking the additional pre- and post- $\mathrm{d} 2$ Tests of Attention, which were administered in all three groups at pre- and post-test data collection.

2.4. Data Collection. On October 21 students in the Fitbit$\mathrm{O}$ and CBPA group and on October 26 students in the comparison group began the pre-test. Prior to the pre-test, the first author explained and showed how to take the d2 Test based on the standardized testing directions and then asked the student to practice the two lines of the test provided on the standardized test directions to ensure that all students understand the testing procedures. Once the students completed the $\mathrm{d} 2$ Test of Attention, students in the two intervention groups were given their Fitbits and were encouraged to wear them Monday through Friday for four weeks. On November 17 students in the Fitbit-O and CBPA group and on November 26 the students in the comparison group took the post-test while following the same testing procedures as the pre-test as well.

D2 Test of Attention. Prior to the beginning of this study, teachers were consulted about what they would like to see their students being tested. Teachers noted that minimizing the amount of time spent on testing and showing gains in attention and concentration are important factors to be considered. Accordingly, the $\mathrm{d} 2$ Test of Attention was chosen. It is easy for students to understand how to take the test given the standardized directions for the test. Also, students will take 4.67 minutes to complete the test. The $\mathrm{d} 2$ Test is a cancellation test that measures neuropsychology performance of the students in the areas of sustained and selective attention as well as concentration. The $\mathrm{d} 2$ Test allows participants 20 seconds per line to selectively identify the letter " $d$ " with two dashes, either above, below, or one dash on top and one on the bottom. Distractors come in two forms, more or less dashes above or below the " $\mathrm{d}$ " and the letter "p" [33].

$$
\text { d d d }
$$

The $\mathrm{d} 2$ Test offers five main outcomes (parameters): (a) the total number of items processed (TN) (i.e., processing speed); (b) the total number of symbols processed minus the total number of errors (TN-E) (i.e., focused attention); (c) the total number of correct responses minus commission errors (CP) (i.e., concentration performance); it measures the ability to attend to stimuli while disregarding other irrelevant tasks; (d) the percentage of all errors (E\%), in which omission and commission are made within all items processed (i.e., accuracy); and (e) fluctuation rate (FR): it is determined by subtracting the line with the lowest number of symbols process from the line with the highest number of symbols process (i.e., sustained attention) [33]. The $\mathrm{d} 2 \mathrm{Test}$ had high test-retest reliability coefficients for all parameters, ranging from .95 to .98 [33]. The $\mathrm{d} 2$ Test has been proven to be an internally valid measure of scanning accuracy, speed, discriminant validity, and fluctuation across trials [34].

2.5. Data Analysis. TN, TN-E, CP, E\%, and FR were used as parameters of attention and concentration performance for data analysis of the $\mathrm{d} 2$ Test based upon the guidelines set by Brickenkamp et al. [33]. Descriptive statistics of $\mathrm{TN}, \mathrm{TN}-\mathrm{E}, \mathrm{CP}, \mathrm{E} \%$, and $\mathrm{FR}$ at pre-test and post-test were conducted for each group. A $2 \times 3$ analysis of variance (ANOVA) was conducted with the time (pre-test versus posttest) as a within-subjects factor and treatment conditions (CBPA, Fitbit-O, and control) as a between-subjects factor. The repeated measure ANOVAs were conducted separately for the TN, TN-E, CP, E\%, and FR. Subsequently, a post hoc comparison was performed to examine the mean difference in each dependent variable between groups (i.e., CBPA-Bs versus Fitbit-O; CBPA-Bs versus control; and Fitbit-O versus control) from pre- to post-test. All statistical analyses were conducted with IBM SPSS version 24 and a significant level of $p<.05$ was set.

\section{Results}

3.1. Preliminary Analysis. Table 1 presents the descriptive statistics of pre- and post-test in d2 Test of Attention among the three groups. At pre-test, the control group's mean scores in processing speed (TN), focused attention (TN-E), and concentration performance $(\mathrm{CP})$ were higher than the two intervention groups. The higher numbers of TN, TN-E, and $\mathrm{CP}$ indicate the better performance in attention and concentration. The control group's mean score in accuracy (E\%) was similar to the CBPA's mean score and lower than the Fitbit-O's mean score. The control group's mean score in attention span (FR) was similar to the Fitbit-O's mean score and lower than the CBPA group's mean score. The lower scores in E\% and FR represent the better accuracy and attention span. Further, 
TABLE 1: Descriptive statistics of pre- and posttest among CBPA, Fitbit-O, and control groups.

\begin{tabular}{|c|c|c|}
\hline & $\begin{array}{l}\text { Pretest } \\
M \pm S D\end{array}$ & $\begin{array}{l}\text { Posttest } \\
\mathrm{M} \pm \mathrm{SD}\end{array}$ \\
\hline \multicolumn{3}{|l|}{ CBPA $(n=31)$} \\
\hline Processing Speed (TN) & $271.68 \pm 38.99$ & $362.65 \pm 76.27$ \\
\hline Focused Attention (TN-E) & $250.84 \pm 41.81$ & $353.03 \pm 77.16$ \\
\hline Concentration Performance (CP) & $93.65 \pm 26.57$ & $138.68 \pm 29.68$ \\
\hline Accuracy (Error \%) & $7.15 \pm 7.72$ & $2.74 \pm 3.55$ \\
\hline Attention Span (FR) & $17.32 \pm 7.02$ & $14.39 \pm 7.19$ \\
\hline \multicolumn{3}{|l|}{ Fitbit-O $(n=28)$} \\
\hline Processing Speed (TN) & $278.79 \pm 49.46$ & $357.14 \pm 73.36$ \\
\hline Focused Attention (TN-E) & $257.29 \pm 48.28$ & $336.86 \pm 67.66$ \\
\hline Concentration Performance (CP) & $94.11 \pm 25.23$ & $109.93 \pm 33.95$ \\
\hline Accuracy (Error \%) & $8.96 \pm 9.06$ & $6.47 \pm 9.24$ \\
\hline Attention Span (FR) & $14.86 \pm 5.82$ & $18.90 \pm 9.89$ \\
\hline \multicolumn{3}{|l|}{ Control $(n=55)$} \\
\hline Processing Speed (TN) & $317.7 \pm 66.37$ & $374.18 \pm 82.18$ \\
\hline Focused Attention (TN-E) & $293.02 \pm 61.67$ & $350.55 \pm 82.83$ \\
\hline Concentration Performance (CP) & $112.58 \pm 34.04$ & $133.00 \pm 34.95$ \\
\hline Accuracy (Error \%) & $7.19 \pm 10.01$ & $4.34 \pm 7.83$ \\
\hline Attention Span (FR) & $14.77 \pm 7.27$ & $17.87 \pm 8.54$ \\
\hline
\end{tabular}

TABLE 2: Results of the repeated measure ANOVA.

\begin{tabular}{|c|c|c|c|c|c|}
\hline Dependent Variables & Factors & $\mathrm{F}$ & $\mathrm{df}$ & $\mathrm{P}$ & $\eta^{2}$ \\
\hline \multirow[t]{2}{*}{ Processing Speed (TN) } & Time & 140.52 & 1 & .000 & .561 \\
\hline & Time $*$ Treatment & 3.372 & 2 & .038 & .058 \\
\hline \multirow[t]{2}{*}{ Focused Attention (TN-E) } & Time & 193.44 & 1 & .000 & .637 \\
\hline & Time $*$ Treatment & 4.37 & 2 & .015 & .074 \\
\hline \multirow[t]{2}{*}{ Concentration Performance (CP) } & Time & 160.14 & 1 & .000 & .593 \\
\hline & Time $*$ Treatment & 13.53 & 2 & .000 & .197 \\
\hline \multirow[t]{2}{*}{ Accuracy (Error \%) } & Time & 21.35 & 1 & .000 & .163 \\
\hline & Time $*$ Treatment & .194 & 2 & .824 & .004 \\
\hline \multirow[t]{2}{*}{ Attention Span (FR) } & Time & 2.71 & 1 & .102 & 024 \\
\hline & Time $*$ Treatment & 8.04 & 2 & .001 & .024 \\
\hline
\end{tabular}

regarding the pre-test, independent sample t-tests revealed no significant mean differences between CBPA and FitbitO groups in TN, TN-E, CP, E\%, and FR. Similarly, there is no significant mean difference in E\% and FR between CBPA group and the control group. However, the t-tests showed that the control group exhibited significantly higher scores in $\mathrm{TN}$, TN-E, and CP than the CBPA group $(\mathrm{t}=4.058, p=.000 ; \mathrm{t}=$ $3.571, p=.001 ; \mathrm{t}=2.944, p=.002)$.

Regarding the changes from pre- to post-test scores for each group, all three groups showed improvement in mean scores of TN, TN-E, CP, and E\% compared to the pre-test. The CBPA group's mean score in FR decreased from the pretest, while both Fitbit-O group's and the control group's mean score of FR increased compared to the pre-test. Across the three groups, the CBPA group's mean scores in TN-E and CP were higher and mean score in $\mathrm{E} \%$ was lower than the two groups; the control group's mean scores in TN-E and CP were higher and mean score in E\% was lower than the Fitbit-O group. However, the CBPA group's mean scores in TN and FR were lower than the control group, but higher than the Fitbit-O group.

3.2. Results of Repeated Measure ANOVA. Table 2 illustrates the results of the repeated measure ANOVA. The results showed significant main effect of time in processing speed (TN) $\left(F_{1}=140.52, p=.000, \eta^{2}=0.561\right)$, focused attention (TNE) $\left(F_{1}=193.44, p=.000, \eta^{2}=0.637\right)$, concentration performance $(\mathrm{CP})\left(F_{1}=160.14, p=.000, \eta^{2}=0.593\right)$, and accuracy $(\mathrm{E} \%)\left(F_{1}=21.35, p=.000, \eta^{2}=0.163\right)$, but not in FR. The three groups showed significant improvement in processing speed, focused attention, concentration performance, and accuracy from pre- to post-test.

Further, as presented in Table 2, the repeated measure ANOVA revealed a significant interaction between time $\times$ 
TABLE 3: Results of post hoc comparisons between groups in five dependent variables.

\begin{tabular}{|c|c|c|c|c|}
\hline Dependent Variables & Between Groups & $F$ & $d f$ & $P$ \\
\hline \multirow[t]{3}{*}{ Processing Speed (TN) } & CBPA vs. Fitbit-O & .925 & 1 & .340 \\
\hline & CBPA vs. Control & 6.876 & 1 & .010 \\
\hline & Fitbit-O vs. Control & 1.842 & 1 & .178 \\
\hline \multirow[t]{3}{*}{ Focused Attention (TN-E) } & CBPA vs. Fitbit-O & 1.73 & 1 & .194 \\
\hline & CBPA vs. Control & 10.688 & 1 & .002 \\
\hline & Fitbit-O vs. Control & 1.874 & 1 & .175 \\
\hline \multirow[t]{3}{*}{ Concentration performance (CP) } & CBPA vs. Fitbit-O & 24.162 & 1 & .000 \\
\hline & CBPA vs. Control & 26.454 & 1 & .000 \\
\hline & Fitbit-O vs. Control & .001 & 1 & .976 \\
\hline \multirow[t]{3}{*}{ Accuracy (Error \%) } & CBPA vs. Fitbit-O & .538 & 1 & .466 \\
\hline & CBPA vs. Control & .297 & 1 & .587 \\
\hline & Fitbit-O vs. Control & .001 & 1 & .976 \\
\hline \multirow[t]{3}{*}{ Attention Span (FR) } & CBPA vs. Fitbit-O & 6.891 & 1 & .011 \\
\hline & CBPA vs. Control & 14.090 & 1 & .000 \\
\hline & Fitbit-O vs. Control & 0.568 & 1 & .453 \\
\hline
\end{tabular}

treatment in processing speed $(\mathrm{TN})\left(F_{2}=3.372, p=.038\right.$, $\left.\eta^{2}=0.058\right)$, focused attention (TN-E) $\left(F_{2}=4.37, p=.015\right.$, $\left.\eta^{2}=0.074\right)$, concentration performance (CP) $\left(F_{2}=13.53\right.$, $\left.p=.000, \eta^{2}=0.197\right)$, and attention $\operatorname{span}(\mathrm{FR})\left(F_{2}=\right.$ $\left.8.04, p=.001, \eta^{2}=0.128\right)$, but not in accuracy (E\%). The results indicated that significant changes in processing speed, focused attention, concentration performance, and attention span were associated with within subject factors (from preto post-test) and between subject factors (three groups), but not related to accuracy.

3.3. Results of Post Hoc Comparisons. Table 3 shows the post hoc comparisons between two groups from pre- to post-test. The results indicated that there were significant differences in concentration performance (CP) $\left(F_{1}=24.162, p=.000\right)$ and attention span (FR) $\left(F_{1}=6.891, p=.011\right)$ between the CBPA and the Fitbit-O group from pre- to post-test, but not in TN, TN-E, and E\%. This means that students in the CBPA were better able to concentrate and sustain their attention after the intervention compared to Fitbit-O group. Further, significant changes between the CBPA group and the control group were found in processing speed (TN) $\left(F_{1}\right.$ $=6.876, p=.010)$, focused attention (TN-E) $\left(F_{1}=10.688, p\right.$ $=.002)$, concentration performance $(\mathrm{CP})\left(F_{1}=26.46, p=\right.$ $.000)$, and attention span (FR) $\left(F_{1}=14.090, p=.000\right)$, but not in accuracy $(\mathrm{E} \%)$. The results indicated that students in the CBPA significantly increased their scores in TN, TN-E, $\mathrm{CP}$, and FR over the control group. In contrast, no significant changes in all five parameters were found between the Fitbit$\mathrm{O}$ and the control groups.

\section{Discussion}

This study was central to examining whether students who participated in coordinated-bilateral PA breaks showed a greater increase from pre- to post-test in attention and concentration than students in the Fitbit- $\mathrm{O}$ and the control groups. In line with the results of previous studies [2, 28], the students in the CBPA group demonstrated a significantly greater increase from pre- to post-test on the $\mathrm{d} 2$ Test than the students in the Fitbit-O and the control groups. Largely supporting the hypothesis, the results indicated that the 4week, daily 6-minute coordinated-bilateral PA breaks in a classroom significantly improved children's processing speed (TN), focused attention (TN-E), concentration performance (CP), and attention span (FR), compared to the Fitbit-O and the control groups.

The CBPA was designed to facilitate the exchange of information between the cerebellum and prefrontal cortex by focusing on the movement sequences $[29,30]$. The CBPA was accomplished by using timed sequences, repetitive patterned movements, and contralateral movements to engage both hemispheres of the brain [28]. Further, the present results supported that "motor development and cognitive development may be fundamentally related" [29] (p.44). This study supported the notion that highly focused, coordinatedbilateral activities in short increments improved attention and concentration through motor development without the need to add an academic component [2, 29-31]. The CBPA was found to be a feasible alternative to academic lessons, while still providing a PA break for the classroom.

The uniqueness of this study was that the 4-week, daily 6-minute CBPA breaks showed pronounced improvement in processing speed (TN), focused attention (TN-E), concentration performance (CP), and attention span (FR). Previous studies examined the acute effect of a single bout of aerobic coordinated movement on attention assessed with $\mathrm{d} 2$ Test $[2,15,28]$. Budde et al. [2] found significant improvements in processing speed (TN), focused attention (TN-E), and accuracy $(\mathrm{E} \%)$. But, they did not found improvement in concentration performance (CP) and attention span (FR). Schmidt et al. [28] reported significant increases in processing speed $(\mathrm{TN})$, accuracy $(\mathrm{E} \%)$, and concentration performance $(\mathrm{CP})$, but not in focused attention (TN-E) and attention span 
(FR). This study indicated that the addition of short CBPA breaks into the classroom routine helped children sustain and sharpen their attention. By helping children find a way to better attend to their learning, schools may be able to reduce further problems of self-control and thus improve academic outcomes for all students [2, 28, 31].

Also, it is important to note that there were significant differences in concentration performance $(\mathrm{CP})$ and attention span (FR) between the CBPA and Fitbit-O group, but no significant differences in the five parameters between the Fitbit$\mathrm{O}$ and the control group. This adds merit to the idea that adding highly focused, coordinated-bilateral activities to the school day can bring about positive changes in concentration and sustained attention, but the non-focused PA may not. However, the results did reveal that the non-focused PA may potentially affect a student's processing speed (TN), focused attention (TN-E), and accuracy (E\%). Supporting the result, Schmidt et al. [28] found the most significant improvement in attention and concentration came from students' engaging in highly aerobic activities. Similarly, Gallotta et al. [15] found improvements in attention and concentration with aerobic activity and any type of breaks, compared no breaks. Future studies may consider adding both highly focused, sequentially coordinated exercises and aerobic activities to academic lessons to see if there is a significant difference between these strategies.

This study highlights three reasons why using the CBPA strategies is a feasible option for classrooms. First, they do not require additional space. Unlike many of the other studies, this study did not use the gym, playground, or hallways. Instead, children were able to stand next to their desks to complete the activities. Secondly, no additional teachers' training in CBPA breaks or researchers were needed to facilitate the CBPA breaks. In contrast, other classroombased PA breaks are required in-service training for the teachers [22, 23]. Finally, the interruption to the child's academic learning was minimal, only six minutes. In short, students who participated in the 4-week, daily 6-minute CBPA breaks without any additional academic component showed improvements in attention and concentration.

It is important to note that this study has four limitations. The first limitation was related to potential confounders (e.g., IQ and SES) that may influence the children's attentional performance. Although this study did not directly collect the information from the students, the participating students who enrolled in the two participating schools came from ethnic and racial diverse and low-income neighborhood. The second limitation is that the study was conducted in schools which made it difficult to do the random assignment. We used available schools as the participating schools (convenient samples) and assigned the schools to either the experimental or the control condition based on their preference. This may account for the higher baseline $\mathrm{d} 2$ scores found in the control school. The third limitation of the study which is related to the students' use of the Fitbit Challenge may also have impacted the outcomes for the $\mathrm{d} 2$ test, in addition to engaging in the 4week, daily 6-minute CBPA breaks. However, the differences between the two intervention groups suggested that the Fitbit itself did not significantly alter the results. The likely increase in PA which was brought about by the Fitbit Challenge may account for the differences on the $\mathrm{d} 2$ Test between the CBPA group and the Fitbit-O group and the control group. Additionally, both intervention groups were meant to take part in the Fitbit Challenge, but the teacher from the Fitbit$\mathrm{O}$ group opted out of this part of the study. Therefore, the impact of the coordinated-bilateral activities cannot be said to be solely responsible for the differences in the $\mathrm{d} 2 \mathrm{Test}$. The last limitation was associated with no emphasis placed on increasing aerobic activity in this intervention. So, there was no direct comparison which could be made between aerobic and coordinated-bilateral activities. Future studies may add aerobic-typed PA to the coordinated-bilateral activities for running the classroom-based PA breaks.

It was concluded that students who participated in 4-week, daily 6-minute CBPA breaks showed significant improvements in processing speed (TN), focused attention (TN-E), concentration performance (CP), and attention span (FR), compared to the control group. The unique result was that the 4-week, daily highly focused, coordinatedbilateral activities showed the significant intervention effects on improving concentration performance and attention span, especially compared to the students who merely focused on increasing their PA levels. This study suggests that engaging students in daily, highly focused, coordinated-bilateral activities is an effective strategy to improve attention and concentration in school-aged children.

\section{Disclosure}

The authors declare that the funding source (Hartwig Foundation) had no input into the study design, data collection, analysis and interpretation of data, and the writing of the report or the decision to submit the paper for publication.

\section{Conflicts of Interest}

The authors declare that there are no conflicts of interest.

\section{Acknowledgments}

The authors would like to thank the teachers, children, and the district for participating in this project.

\section{References}

[1] National Institute of Mental Health (NIMH), "Attention Deficit Hyperactivity Disorder 2015,” 2016, http://www.nimh.nih.gov/ health/topics/attention-deficit-hyperactivity-disorder-adhd/ index.shtml.

[2] H. Budde, C. Voelcker-Rehage, S. Pietraßyk-Kendziorra, P. Ribeiro, and G. Tidow, "Acute coordinative exercise improves attentional performance in adolescents," Neuroscience Letters, vol. 441, no. 2, pp. 219-223, 2008.

[3] J. B. Bartholomew and E. M. Jowers, "Physically active academic lessons in elementary children," Preventive Medicine, vol. 52, pp. s51-s54, 2011.

[4] M. C. Davidson, D. Amso, L. C. Anderson, and A. Diamond, "Development of cognitive control and executive functions 
from 4 to 13 years: evidence from manipulations of memory, inhibition, and task switching," Neuropsychologia, vol. 44, no. 11, pp. 2037-2078, 2006.

[5] R. Bull and G. Scerif, "Executive functioning as a predictor of children's mathematics ability: Inhibition, switching, and working memory," Developmental Neuropsychology, vol. 19, no. 3, pp. 273-293, 2001.

[6] Y. Ahamed, H. Macdonald, K. Reed, P. J. Naylor, T. LiuAmbrose, and H. Mckay, "School-based physical activity does not compromise childrens academic performance," Medicine \& Science in Sports \& Exercise, vol. 39, no. 2, pp. 371-376, 2007.

[7] R. Elliott, "Executive functions and their disorders," British Medical Bulletin, vol. 65, pp. 49-59, 2003.

[8] C. R. Greenwood, J. C. Delquadri, and R. V. Hall, "Longitudinal Effects of Classwide Peer Tutoring," Journal of Educational Psychology, vol. 81, no. 3, pp. 371-383, 1989.

[9] S. N. Blair, "Physical inactivity: the biggest public health problem of the 21st century," British Journal of Sports Medicine, vol. 43, no. 1, pp. 1-2, 2009.

[10] S. C. Dumith, D. P. Gigante, M. R. Domingues, and H. W. Kohl, "Physical activity change during adolescence: A systematic review and a pooled analysis," International Journal of Epidemiology, vol. 40, no. 3, Article ID dyq272, pp. 685-698, 2011.

[11] R. Lowry, S. M. Lee, J. E. Fulton, and L. Kann, "Healthy people 2010 objectives for physical activity, physical education, and television viewing among adolescents: National trends from the youth risk behavior surveillance system, 1999-2007," Journal of Physical Activity \& Health, vol. 6, no. 1, pp. S36-S45, 2009.

[12] J. F. Sallis, J. J. Prochaska, and W. C. Taylor, "A review of correlates of physical activity of children and adolescents," Medicine \& Science in Sports \& Exercise, vol. 32, no. 5, pp. 963$975,2000$.

[13] D. M. Castelli, C. H. Hillman, S. M. Buck, and H. E. Erwin, "Physical fitness and academic achievement in third- and fifthgrade students," Journal of Sport \& Exercise Psychology, vol. 29, no. 2, pp. 239-252, 2007.

[14] M. C. Gallotta, G. P. Emerenziani, E. Franciosi, M. Meucci, L. Guidetti, and C. Baldari, "Acute physical activity and delayed attention in primary school students," Scandinavian Journal of Medicine \& Science in Sports, vol. 25, no. 3, pp. e331-e338, 2015.

[15] M. C. Gallotta, L. Guidetti, E. Franciosi, G. P. Emerenziani, V. Bonavolontà, and C. Baldari, "Effects of varying type of exertion on children's attention capacity," Medicine \& Science in Sports \& Exercise, vol. 44, no. 3, pp. 550-555, 2012.

[16] P. D. Tomporowski, C. L. Davis, P. H. Miller, and J. A. Naglieri, "Exercise and children's intelligence, cognition, and academic achievement," Educational Psychology Review, vol. 20, no. 2, pp. $111-131,2008$.

[17] F. Trudeau and R. J. Shephard, "Relationships of Physical Activity to Brain Health and the Academic Performance of Schoolchildren," American Journal of Lifestyle Medicine, vol. 4, no. 2, pp. 138-150, 2010.

[18] D. L. Katz, D. Cushman, J. Reynolds et al., "Putting physical activity where it fits in the school day: Preliminary results of the ABC (Activity Bursts in the Classroom) for fitness program," Preventing Chronic Disease, vol. 7, no. 4, article no. A82, 2010.

[19] G. Mura, M. Vellante, A. E. Nardi, S. Machado, and M. G. Carta, "Effects of school-based physical activity interventions on cognition and academic achievement: A systematic review," CNS and Neurological Disorders - Drug Targets, vol. 14, no. 9, pp. 1194-1208, 2015.
[20] C. N. Rasberry, S. M. Lee, L. Robin et al., "The association between school-based physical activity, including physical education, and academic performance: A systematic review of the literature," Preventive Medicine, vol. 52, pp. S10-S20, 2011.

[21] P. D. Tomporowski, K. Lambourne, and M. S. Okumura, "Physical activity interventions and children's mental function: an introduction and overview," Preventive Medicine, vol. 52, pp. S3-S9, 2011.

[22] J. E. Donnelly, J. L. Greene, C. A. Gibson et al., "Physical Activity Across the Curriculum (PAAC): a randomized controlled trial to promote physical activity and diminish overweight and obesity in elementary school children," Preventive Medicine, vol. 49, no. 4, pp. 336-341, 2009.

[23] M. T. Mahar, S. K. Murphy, D. A. Rowe, J. Golden, A. T. Shields, and T. D. Raedeke, "Effects of a classroom-based program on physical activity and on-task behavior," Medicine \& Science in Sports \& Exercise, vol. 38, no. 12, pp. 2086-2094, 2006.

[24] L. A. Grieco, E. M. Jowers, and J. B. Bartholomew, "Physically active academic lessons and time on task: The moderating effect of body mass index," Medicine \& Science in Sports \& Exercise, vol. 41, no. 10, pp. 1921-1926, 2009.

[25] J. E. Donnelly and K. Lambourne, "Classroom-based physical activity, cognition, and academic achievement," Preventive Medicine, vol. 52, pp. S36-S42, 2011.

[26] E. K. Howie, M. W. Beets, and R. R. Pate, "Acute classroom exercise breaks improve on-task behavior in 4th and 5th grade students: A dose-response," Mental Health and Physical Activity, vol. 7, no. 2, pp. 65-71, 2014.

[27] J. K. Ma, L. L. Mare, and B. J. Gurd, "Four minutes of in-class high-intensity interval activity improves selective attention in 9to 11-year olds," Applied Physiology, Nutrition, and Metabolism, vol. 40, no. 3, pp. 238-244, 2015.

[28] M. Schmidt, F. Egger, and A. Conzelmann, "Delayed positive effects of an acute bout of coordinative exercise on children's attention," Perceptual and Motor Skills, vol. 121, no. 2, pp. 431446, 2015.

[29] A. Diamond, "Close interrelation of motor development and cognitive development and of the cerebellum and prefrontal cortex," Child Development, vol. 71, no. 1, pp. 44-56, 2000.

[30] S. Pedersen, "Deliberate laterality practice facilitates sensorymotor processing in developing children," Physical Education and Sport Pedagogy, vol. 19, no. 2, pp. 136-148, 2014.

[31] I. M. J. van der Fels, S. C. M. te Wierike, E. Hartman, M. T. Elferink-Gemser, J. Smith, and C. Visscher, "The relationship between motor skills and cognitive skills in 4-16 year old typically developing children: A systematic review," Journal of Science and Medicine in Sport, vol. 18, no. 6, pp. 697-703, 2015.

[32] E. McClelland, A. Pitt, and J. Stein, "Enhanced academic performance using a novel classroom physical activity intervention to increase awareness, attention and self-control: Putting embodied cognition into practice," Improving Schools, vol. 18, no. 1, pp. 83-100, 2015.

[33] R. Brickenkamp, The d2 Test of Attention 10th Expanded and Revised Edition, Hogrefe Publishers, 2002.

[34] M. E. Bates and E. P. Lemay Jr., "The d2 test of attention: construct validity and extensions in scoring techniques," Journal of the International Neuropsychological Society, vol. 10, no. 3, pp. 392-400, 2004. 